the quantitative tools: Patient Specific Functional Score (PSFS) self-reporting of functional ability to complete tasks and the Numerical Pain Rating Scale (NPRS) self-rating of pain score. A cross-sectional analysis of absenteeism rates was also performed to determine the percentage of WBP participants with certified absence and work hours lost for back pain.

Result In 2012, 75\% of participants found the WBP beneficial. This figure rose to $96 \%$ and $94 \%$ in 2015 and 2016 respectively.

In 2012, 52\% of participants achieved a 2 point increase or greater for their average score in the PSFS. In 2015 and 2016 it was $88 \%$ and $72 \%$ respectively. A 2 point change for the average score is valid to be $90 \%$ confident that a real improvement occurred.

In $201266 \%$ reported a reduction in pain levels on the NPRS. In 2015 and 2016 this increased to $96 \%$ and 92\%, respectively.

In 2012, 52\% of the WBP participants had some certified leave of absence for back pain. This decreased to $21 \%$ in 2015 and to $12 \%$ in 2016.

Work hours lost for back pain in 2012 was $2.5 \%$ of the total percent of absenteeism. This decreased in 2015 and in 2016 to $0.4 \%$ and $0.6 \%$ respectively (a reduction of $76 \%$ in back related absence in a four year period).

Discussion The WBP demonstrated both personal and organisational benefits. It has proven to be a worthwhile health promotion initiative. It's ethos is based on an active approach in the management of back pain, enabling staff to remain at work while effectively managing back pain.

\section{BARRIERS AND WORK IMPAIRMENT ASSOCIATED TO SICKNESS PRESENCE}

A Skerjanc, MD Fikfak. Clinical Institute for Occupational, Traffic and Sports Medicine, University Medical Centre Ljubljana, Slovenia

\subsection{6/oemed-2018-ICOHabstracts.979}

Introduction Sickness presence is a relatively new 'phenomenon that people, despite complaints and ill health that should prompt them to rest and take sick leave, go to work in any case'. The highest sickness presence is largely to be found in the care and welfare and educational sectors. The aim of the study is to investigate the relations between barriers and work impairment and sickness presence among health care workers.

Methods A cross-sectional study was conducted at the largest hospital in Slovenia involving 5865 health care workers employed at the University Medical Centre Ljubljana in the year 2010. Each employee obtained a questionnaire composed of three standardised international questionnaires. Logistic regression methods were used to assess the associations between risk factors and sickness presence. The questionnaire contained questions related to demographic factors and factors that describe one's own assessment of workability (absenteeism, barriers and work impairment, anticipated certainty about one's own workability in the following year). Data was analysed using SPSS.

Results In multivariate modelling the highest odds were calculated for barriers at work $(\mathrm{OR}=4,5 ; 95 \% \mathrm{CI}: 3,8$ to 5,3$)$, anticipated uncertainty about workability $(\mathrm{OR}=1,9 ; 95 \% \mathrm{CI}$ : $1,5$ to 2,3$)$, sickness absence more than twice a year $(\mathrm{OR}=3,6 ; 95 \% \mathrm{CI}: 3,0$ to 4,4$)$ and sickness absence more than twice in the past year $(\mathrm{OR}=2,7 ; 95 \% \mathrm{CI}: 2,2$ to 3,3$)$.
Conclusion This is the first surveillance on sickness presence indicates that besides the pre-requisite - bad health, sickness presence is associated with barriers at work, their consequences on behaviour of the workers and sickness absence. If continued they might have negative consequences on work and health care worker's health and require a response in health care organisation.

\section{TITLE CORRELATES OF PRESENTEEISM: A STUDY OF NURSES WORKING IN GERIATRIC SETTINGS IN MALTA}

L Fiorini* ${ }^{*}$ A Griffiths, J Houdmont. Institute of Mental Health. University of Nottingham, Nottingham, UK

\subsection{6/oemed-2018-ICOHabstracts.980}

Introduction Presenteeism is usually defined as attending for work while ill. It is linked with lost productivity and increased sickness absence, and can be costly to organisations. Studies suggest a high prevalence of presenteeism in the healthcare sector. Additionally European statistics suggest that presenteeism is particularly frequent in Malta (EU). A study was therefore conducted to investigate the correlates of presenteeism in a sample of nurses working within a geriatric ward setting in Malta.

Methods A cross-sectional survey $(n=270)$ investigated the suggested predictors of presenteeism that had emerged in an earlier qualitative study. Hierarchical binary logistic regression was used to identify the correlates of presenteeism. Data was analysed using SPSS.

Result Individuals who had engaged in presenteeism two or more times in the previous 12 months were more likely to have: engaged in sickness absenteeism frequently (OR 2.36, 95\% CI: 1.02 to 5.94); felt emotional during their last presenteeism episode (OR 1.21, 95\% CI: 1.07 to 1.37); felt that their last sickness absenteeism episode was good for their health (OR 1.72, 95\% CI: 1.14 to 2.61); and felt presenteeism was necessary following recent sick leave (OR 1.45, 95\% CI: 1.08 to 1.94$)$. They were also less likely to have: reported a fracture (OR $0.06,95 \% \mathrm{CI}: 0.01$ to 0.63 ) or gastric illness (OR $0.35,95 \% \mathrm{CI}: 0.15$ to 0.82 ) during their last absenteeism episode; understood the illness that led to their last presenteeism episode (OR 0.80, 95\% CI: 0.67 to 0.95 ); and to feel that they had managerial and peer support (OR $0.45,95 \%$ CI: 0.26 to 0.91 ).

Discussion The study highlights that presenteeism is linked to overall health, however perceptions of individual illnesses can also influence the frequency of this behaviour. The study also supports previous findings that work attitudes and organisational factors also play a role. Interventions that benefit nurses' health and provide support at work may reduce the frequency of presenteeism.

\section{DEVELOPMENT OF AN INTERVENTION TO FACILITATE RETURN TO WORK OF UK HEALTHCARE STAFF WITH COMMON MENTAL HEALTH DISORDERS}

${ }^{1}$ Dorota Juszczyk, ${ }^{2}$ Max Henderson, 'Vaughan Parsons, ${ }^{3}$ Julia Smedley, ${ }^{4}$ Robert Shannon, ${ }^{1}$ Ira Madan. 'Guy's And St. Thomas' NHS Foundation Trust, London, UK; ${ }^{2}$ St James University Hospital, Leeds, UK; ${ }^{3}$ Southampton University Hospitals NHS Trust, Southampton, UK; ${ }^{4}$ University of Southampton, Southampton, UK

10.1136/oemed-2018-ICOHabstracts.981 
Introduction The National Health Service (NHS) is the biggest employer in the United Kingdom (UK). Depression and anxiety are the most common reasons for sickness absence in the NHS. As part of a trial feasibility study, we developed an intervention to facilitate an earlier return to work (RTW) in NHS staff absent with common mental health disorders (CMHD).

Methods We used iterative methodology, based on MRC guidance. Evidence was sought from systematic reviews, guidelines, and work known to the research team on the key components of the case-management (Stage 1). During Stage 2, the evidence from Stage 1 was mapped onto the proposed intervention together with input from international experts and key stakeholders.

Results Evidence suggests that an intervention based on a casemanagement model using a biopsychosocial approach could be cost-effective and lead to earlier RTW. In our study, specially trained occupational health nurses will deliver the intervention. Case-management will be conducted during regular consultations (every 2 to 4 weeks). Key components will include: identifying obstacles to RTW, collaborative problem solving based on cognitive behaviour principles focussing on work outcomes, work-focused goal setting, development of a RTW plan, and peer support to increase return to work self-efficacy. Work adjustments, work visits or therapeutic RTW will be considered. The case-manager will communicate with the line and human resources managers and treating healthcare professionals after each consultation. A bespoke information leaflet will be developed and given to line managers and workers emphasising the therapeutic importance of early RTW.

Discussion To our knowledge WB2W is the first intervention addressing RTW among UK healthcare staff with CMHDs. A key output from this research will be a complete specification of the intervention package including a manual for training the case managers and practical service information to guide the design of a randomised controlled trial.

\section{SHARPS INJURIES AMONG HEALTH CARE PERSONNEL IN A CHINESE HOSPITAL}

L Xiaoxia, B Adam*. University of Debrecen, Faculty of Public Health, Department of Preventive Medicine, Debrecen, Hungary

\subsection{6/oemed-2018-ICOHabstracts.982}

Introduction Sharps injury is a penetrating wound from a needle, scalpel, or another sharp object that may result in exposure to blood or other body fluids. Sharps injuries constitute a serious occupational health problem for health care personnel and can result in high direct and indirect costs for the health care facility. The aim of the study was to examine the prevalence and characteristics of sharps injuries among Chinese health care workers.

Methods A questionnaire survey was carried out in a Chinese hospital to collect demographic and occupational data, information on sharps injuries and their reporting. Multiple logistic regression was used to analyse the potential risk factors of sharps injuries, including gender, age, education level, position, department and perception of the safety culture.

Results The 5 year prevalence of sharps injuries was found $41 \%$ among the hospital personnel. Sex, age and education did not influence significantly sharps injuries $(p=0.798$, $\mathrm{p}=0.886$ and $\mathrm{p}=0.47$, respectively). However, the position of staff and especially the department where they work significantly correlated with sustaining such an accident
( $\mathrm{p}=0.025$ and $\mathrm{p}<0.0001$, respectively). $86 \%$ of accidents hurt fingers, $90 \%$ of injuries were sustained in inpatient units. Six sharp devices were responsible for nearly $95 \%$ of all injuries and $86 \%$ of them occurred when health care workers were using the devices. Association could not be described between sharps injuries and the perceived culture of safety. About three-fourth of sharps injuries were not reported.

Discussion A high prevalence rate of sharps injuries was observed in the studied hospital, the majority of injured workers were nurses. The rate varied significantly by department and position. The study found significant under-reporting. Adequate level of occupational health and safety for health care personnel can only be provided with efficient prevention from sharps injuries, which needs information on risk factors and a well-functioning reporting system.

\section{SURVEY OF RESPIRATORY PROTECTION PROGRAM IN IRANIAN HOSPITALS}

${ }^{1} \mathrm{M}$ Honarbakhsh, ${ }^{2} \mathrm{M}$ Jahangiri, ${ }^{2} \mathrm{H}$ Ghaem, ${ }^{3} \mathrm{P}$ Farhadi. ${ }^{1}$ Student Research Committee, School of Health, Shiraz University of Medical Sciences, Shiraz, Iran; ${ }^{2}$ School of Health, Shiraz University of Medical Sciences, Shiraz, Iran; ${ }^{3}$ Industrial Management Department, School of management and Accounting, Allameh Tabataba'i University, Tehran - Iran

\subsection{6/oemed-2018-ICOHabstracts.983}

Introduction In hospitals, Health Care Workers (HCWs) are exposed to a wide range of respiratory hazards, which requires using respiratory protection equipment and implementation of Respiratory Protection Program (RPP). The aim of this cross-sectional study was to investigate the level of RPP implementation in 36 educational hospitals of Fars province, Iran.

Methods A Self-made checklist including nine components of RPP standard were prepared and completed by the occupational hygienists in hospitals. Fuzzy Analytical Hierarchy Process (FAHP) was used to determine the weight coefficient of RPP components. Finally, Respiratory Protection Program Index (RPPI) was developed to calculate the compliance degree of RPP.

Results The results showed that RPP was not fully implemented in the studied hospitals and the highest and lowest RPPI scores were related to training and fit testing, respectively.

Conclusion Effective protection of HCWs against respiratory hazards requires the full implementation of respiratory protection program in the hospitals. This program must be include evaluation of respiratory hazards, appropriate selection and maintenance of respirators, medical evaluation, fit testing, employee training record keeping, and program evaluation. However, the largest gaps in the RPP elements were discovered in the components of fit testing and medical evaluation which requires significant attempts to promote the situation of RPP in the studied hospital.

\section{NATIONAL HEPATITIS B VACCINATION POLICIES FOR HEALTHCARE WORKERS IN MEMBER-STATES OF THE EUROPEAN UNION: AN UPDATED OVERVIEW}

\footnotetext{
${ }^{1,2} \mathrm{~A}$ de Schryver*, ${ }^{2} \mathrm{~T}$ Lambaerts, ${ }^{2} \mathrm{~N}$ Lammertyn. ${ }^{1}$ University of Antwerpen, Epidemiology and Social Medicine, Antwerpen, Belgium; ${ }^{2}$ IDEWE Occupational Health Services, Leuven, Belgium
}

10.1136/oemed-2018-ICOHabstracts.984 